\title{
N-chlorination and Orton Rearrangement of Aromatic Polyamides, Revisited
}

\author{
Giancarlo Barassi* and Thomas Borrmann
}

School of Physical and Chemical Sciences, Victoria University of Wellington, New Zealand

\begin{abstract}
Polyamide membranes are widely used in water desalination. It is known that they suffer degradation due to the presence of free chlorine. This communication shows a detailed chemical reaction mechanism for the $\mathrm{N}$-chlorination and Orton rearrangement of poly ( $m$-phenylene isophthalamide), which is the linear aromatic polyamide component of the commonly used B-9 Permasep $®$ membrane. The $\mathrm{N}$-chlorination of this aromatic polyamide causes the loss of hydrogen bonding. This triggers conformational changes in the polymer; the polymer becomes less rigid and, void spaces open up, which decreases solute rejection and increases water flux. The $\mathrm{N}$-Chlorination reaction is reversible in alkaline media. Therefore, if the polymer is suspected to have come into contact with hypochlorite anions or hypochlorous acid immediate cleaning with sodium hydroxide could reverse the $\mathrm{N}$-chlorination. Conversely, the $\mathrm{N}$-chlorination is acid catalyzed; hence, special care has to be taken during the cleaning stage, when $\mathrm{HCl}$ is used. Furthermore, $\mathrm{N}$-chlorinated aromatic polyamides can undergo an Orton rearrangement, which is also promoted in acidic media, resulting in the formation of ortho- or para-chloro substituted analogues of the aromatic amide moiety. The chloro group causes a strong negative inductive effect weakening the amide bond making it more susceptible to hydrolysis, which eventually produces chain scission.
\end{abstract}

\section{Introduction}

The majority of water desalination plants currently in operation employ membranes in reverse osmosis processes [1]. These membranes undergo degradation processes due to the presence of hypochlorite ions and hypochlorous acid. Several studies have been published in journals related to the field of membrane science discussing the degradation of polyamides by free chlorine released from $\mathrm{HClO}$ and $\mathrm{ClO}$, which usually is explained assuming well-known mechanisms involving a $\mathrm{N}$-chlorination of the amide followed by an intermolecular rearrangement forming ortho-para- substitution of the aromatic ring [2-11] (agregar degradation [1-5]). This topic is of significant interest to researchers in the field of water treatment as polyamide based membranes become irreversibly damaged by hypochlorite decreasing their performance [12]. Although valuable practical results have been collected in different studies for this 'free chlorine degradation' phenomena, most authors are somehow elusive to show and discuss the mechanism by which free chlorine reacts with the polymeric membranes [3-7]. A study done by Sun G et al. [5] showed a reprint from the book 'The Chemistry of Amides' from the PATAI Series [2] for the N-chlorination of N-methyl benzamide. Unfortunately, the figure was not properly transferred and did not state the transition state between the $\mathrm{N}$-methyl benzamide and O-chloroimidate. Furthermore, the study did not show the possible Orton rearrangement leading to the chlorinated species likely to be instrumental in the degradation process. A further study by Cao et al. [6] presented a scheme for $\mathrm{N}$-chlorination followed by Orton rearrangement and chain scission but did not discuss the mechanisms. Additionally, a recent publication in the literature by [13] shows a misleading mechanism in the article's abstract suggesting that the $\mathrm{N}$-chlorination and chain scission occurs in only one step for a fully aromatic polyamide.

Hence it is important to review the original publications in literature for these organic reactions and adapt them to polyamides in order to show a proper equation that will serve as reference for future studies in the field. Furthermore, it is believed that showing and discussing the mechanism will provide valuable insights and suggest possible methods for reducing and avoiding membrane degradation. Consequently, presented here is a revisit of the membrane Degradation process by hypochlorite using and adapting mechanisms for simple compounds from the literature to a polyamide commercially used in membrane filtration systems.

\section{$\mathrm{N}$-chlorination of poly (m-phenylene isophthalamide)}

The N-chlorination of N-methyl benzamide by hypochlorite was studied in depth by Hardy et al. in 1967 [10], who found the reaction to be reversible. Figure 1 shows an adapted version of Hardy's mechanism for poly ( $m$-phenylene isophthalamide). Hardy et al. observed that the reaction of amide with hypochlorite was reversible in alkaline media and proposed a transition state based on a six-membered ring. Values of $2.5 \pm 0$ and $13.7 \pm 1.1 \mathrm{~L} \mathrm{~mol}^{-1} \mathrm{~min}^{-1}$ for the second order rate constant were calculated in his study at $293 \mathrm{~K}$ for the $\mathrm{N}$-chlorination and the basic hydrolysis for the $\mathrm{N}$-chloro $\mathrm{N}$-methyl benzamide, respectively. Furthermore, a raise in the temperature to $303 \mathrm{~K}$ increases the rate constant for the hydrolysis to a value of $47.5 \pm 4.8 \mathrm{~L} \mathrm{~mol}^{-1} \mathrm{~min}^{-1}$. These results show that under alkaline conditions the equilibrium in the reaction between hypochlorite and $\mathrm{N}$-methyl benzamide is displaced towards the reagents. This finding is of significant importance to membrane science as it suggests that polyamide membranes coming into contact with hypochlorite anions or hypochlorous acid could be regenerated using a base treatment. The $\mathrm{N}$-chlorination of the amide moieties in the membrane polymers could potentially be reversed washing the membrane with an alkaline solution. This could be implemented in conjunction with the application of solution of sodium hydroxide used to prevent scaling by colloidal silica on the membrane's

*Corresponding author: Victoria University, Wellington, 6140, New Zealand, PO BOX 600, Tel: +64-4-4635559; E-mail: giancarlo.barassi@vuw.ac.nz

Received March 19, 2012; Accepted May 16, 2012; Published May 21, 2012

Citation: Barassi G, Borrmann T (2012) N-chlorination and Orton Rearrangement of Aromatic Polyamides, Revisited. J Memb Sci Technol 2:115. doi:10.4172/21559589.1000115

Copyright: ( 2012 Barassi G, et al. This is an open-access article distributed under the terms of the Creative Commons Attribution License, which permits unrestricted use, distribution, and reproduction in any medium, provided the original author and source are credited. 
Citation: Barassi G, Borrmann T (2012) N-chlorination and Orton Rearrangement of Aromatic Polyamides, Revisited. J Memb Sci Technol 2:115. doi:10.4172/2155-9589.1000115

Page 2 of 3

surface during cleaning periods. Both membrane degradation due to hypochlorite anion or hypochlorous acid and silica scaling could be prevented in the same membrane cleaning step.

On the other hand, the presence of acid displaces the reaction towards the formation of an O-chloroimidate, which will rapidly form the $\mathrm{N}$-chlorinated product $\left(\mathrm{k}_{3}\right)$. The degradation by hypochlorite of a linear non-aromatic polyamide (nylon 6,6) was studied by Niels Dam et al. [4]. The study concluded that the rate of degradation was $\mathrm{pH}$ dependant, whereby decreasing the $\mathrm{pH}$ increased the $\mathrm{N}$-chlorination and degradation of the polymer, in agreement with the abovementioned suggestion.

Polyamides are known for their high tensile strength which originates from hydrogen bonding between $\mathrm{N}-\mathrm{H}$ and $\mathrm{C}=\mathrm{O}$. $\mathrm{N}$-chlorination causes the polymeric chain to become less rigid due to the loss of the hydrogen bond causing conformational changes in the polymeric chain, which leads to the opening of void spaces. The loss of hydrogen bonding can be followed by IR spectroscopy following the disappearance of the $\mathrm{N}-\mathrm{H}$ stretching vibration peak, for secondary amides, near a wave number of $3300 \mathrm{~cm}^{-1}$ [4]. In the case of membranes used in water purification systems the opening of void spaces will cause higher water flux and/or solute to pass. There have been some attempts reported in the literature on trying to deliberately degrade membranes seeking to increase water flux without decreasing solute rejection. These efforts have been successful as reported by certain manufacturers [12].

\section{Ortonrearrangement of poly ( $m$-phenylenediamine)}

$\mathrm{N}$-chlorinated amides can undergo an Orton rearrangement.
The first observations of this rearrangement using N-halogenated acetanilide were described by Mills in 1860 [9]. Figure 2, shows the proposed mechanism of the Orton rearrangement for the $\mathrm{N}$-chlorinated poly ( $m$-phenylene isophthalamide) obtained from the reaction shown in Figure 1. The rearrangement is intermolecular and occurs in two steps. The first step occurs in acidic media and involves the protonation of the $\mathrm{N}$-chlorinated amide followed by an $\mathrm{S}_{\mathrm{N}} 2$ type mechanism with a nucleophilic attack by chloride from solution on the chlorine bound to the nitrogen. In fact, In gold states that the reaction can be regarded as "a bimolecular nucleophilic substitution by the halide ion at the chlorine atom of a chlor-ammonium ion, a kind of $S_{N} 2$ substitution in an 'onium salt, but at chlorine instead of at carbon" [11]. This reaction generates chlorine gas, which was observed by Orton during his experiments [2]. Moreover, the reaction rates on linear or aromatic polyamides will differ from those for $\mathrm{N}$-chlorinated acetanilide since polymers have decreased rotation and translation compared to those from a molecule decreasing the chance of having an effective collision to undergo an $\mathrm{S}_{\mathrm{N}} 2$ mechanism. The second step involves the electrophilic substitution by chlorine of the aromatic ring. This leads to ortho- or para-substituted chlorinated aromatic polyamide. High yields have been reported for this rearrangement even at room temperature, especially for $\mathrm{N}$-chlorinated amides, which have aromatic functionalities directly adjacent to the chlorinated amide moieties $[8,11]$. Temperature is also a factor to consider. During cleaning stages of RO membranes [12] solutions are commonly used at slightly elevated temperatures of up to $313 \mathrm{~K}$. This means that under acidic and neutral conditions the rate of the Orton rearrangement will be increased noticeably.

In the case of aromatic polyamides, which are prepared using

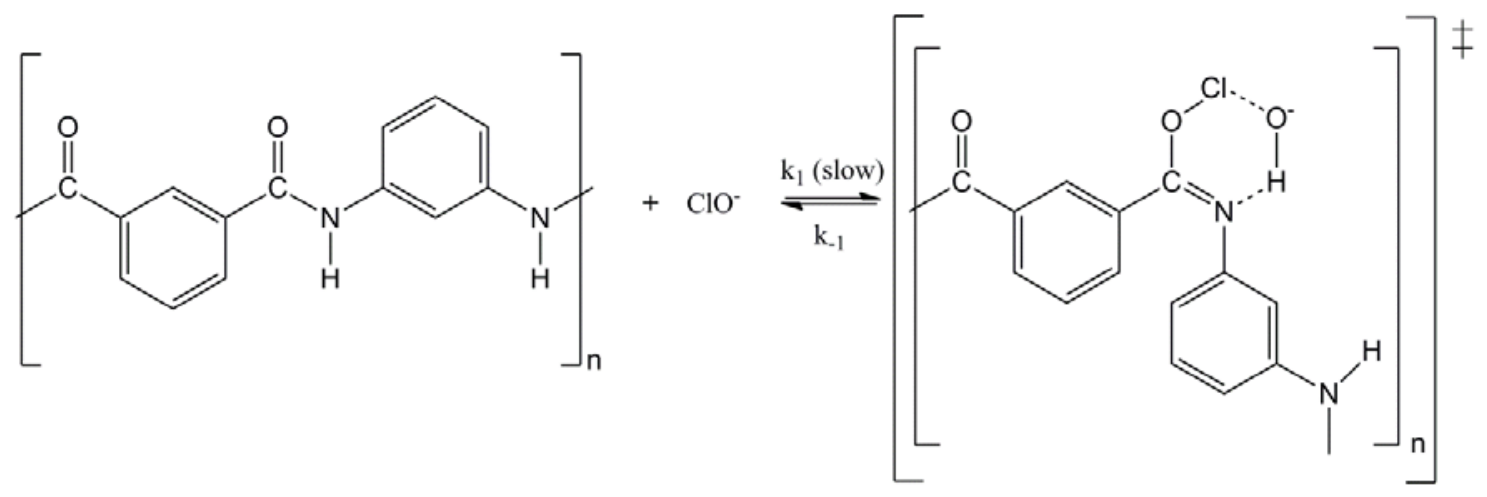

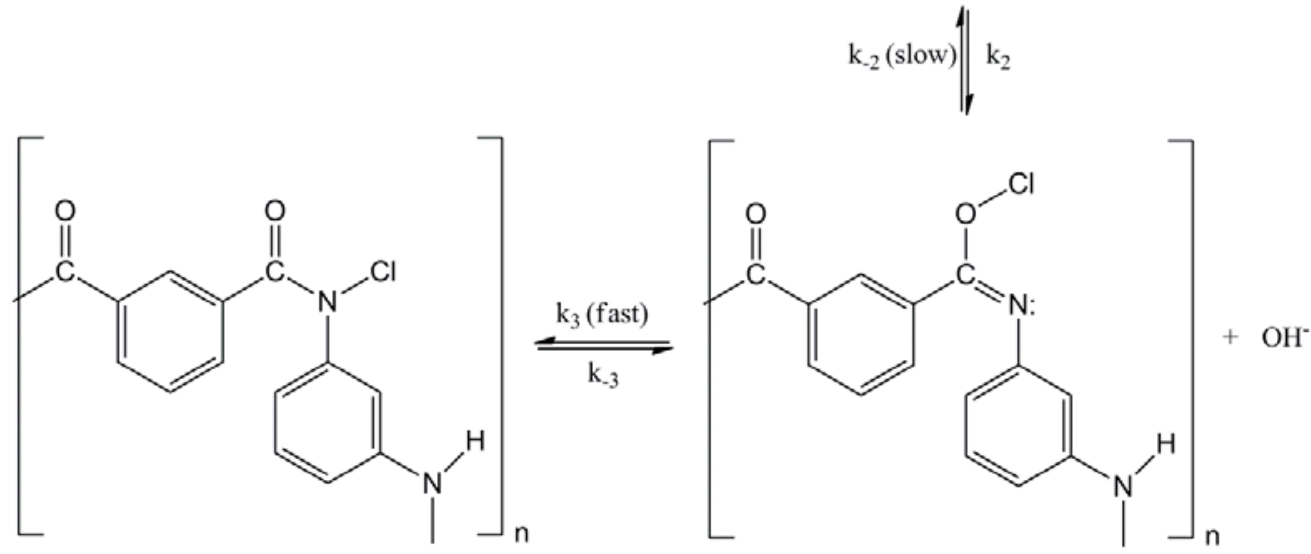

Figure 1: $\mathrm{N}$-chlorination of poly ( $m$-phenylene isophthalamide) by hypochlorite. 


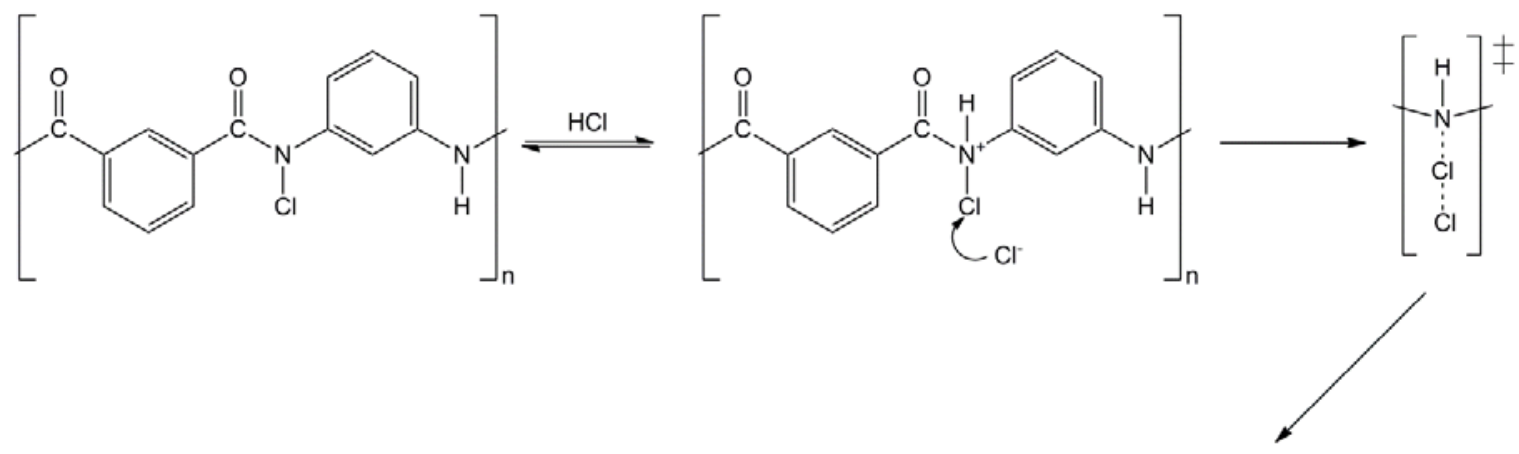<smiles>[CH]N([CH2])c1cccc(NC(=O)c2cccc(C(=O)C(C)(C)C)c2)c1</smiles>

Figure 2: Orton rearrangement of $\mathrm{N}$-chlorinated poly ( $m$-phenylene isophthalamide).

$m$-phenylenediamine as one of the monomers, it is most probable that the free chlorine produced in the Orton rearrangement substitutes hydrogen in the ortho-position to both amide groups attached to the aromatic ring, since this position has the highest electron density.

The Orton rearrangement at a first glance appears to simply restore the amide functionality, allowing hydrogen bonding through the regenerated $\mathrm{N}-\mathrm{H}$ function. However, the chloro-substitution on the aromatic ring causes a negative inductive effect weakening the amide bond of the polymer. This means that, if the polyamide is in presence of an acid or base, it can undergo hydrolysis through the typical mechanism for amides under these conditions.

\section{Conclusions}

The reversibility of the $\mathrm{N}$-chlorination of amides suggests that $\mathrm{N}$-chlorination of polyamides in membranes could be reversed by contacting the polymer with an alkaline solution and, hence, preventing further reactions leading to the degradation of the membrane. Alkaline cleaning of membranes is already undertaken to remove silica precipitates and in light of the reversal of the $\mathrm{N}$-chlorination, it could be considered that alkaline treatment might also prevent further membrane degradation due to free chlorine. It is suggested though that contact times of the membrane with the alkaline could be kept shorter by operating the cleaning solution at $303 \mathrm{~K}$ as the rate constant of the hydrolysis of $\mathrm{N}$-chlorinated $\mathrm{N}$-methyl benzamides is 4 times faster at this temperature than at $293 \mathrm{~K}$. Further studies should be undertaken to elucidate the reaction kinetics and details of the treatment regime required in aromatic polyamides.

Hydrolysis of the N-chlorinated will prevent the occurrence of the Orton rearrangement, an acid catalyzed follow-up reaction, where the free chlorine is generated from the reaction of $\mathrm{N}$-chlorinated amides with chloride from solution. The free chlorine in turn can electrophilically substitute hydrogen on the aromatic rings present in the polymer chains, facilitating the hydrolysis of amide bond sand leading eventually to chain scission.

Both the N-chlorination and Orton rearrangement are promoted in acidic media. Therefore, it is very likely that accelerated membrane degradation will be observed in membrane plant, where chlorination is done as prevention method for biofouling and cleaning is only carried out using hydrochloric acid.

Both the N-chlorination, through the decrease on hydrogen bonding, and the Orton rearrangement, through the eventual hydrolysis of amide bonds, cause an opening of void spaces and, hence, cause a higher water flux and/or solute through the membranes.

\section{References}

1. Wittholz MK, O'Neill BK, Colby CB, Lewis D (2008) Estimating the cost of desalination plants using a cost database. Desalination 229: 10-20.

2. Zabicky J (1970) The chemistry of amides. Interscience, London, New York.

3. Glater J, Hong SK, Elimelech M (1994) The Search for a Chlorine-Resistant Reverse-Osmosis Membrane. Desalination 95: 325-345.

4. Ogilby PR, Dam N (2001) On the mechanism of polyamide degradation in chlorinated water. Helv Chim Acta 84: 2540-2549.

5. Sun G, Sun YY (2004) Novel refreshable N-halamine polymeric biocides: $\mathrm{N}$-chlorination of aromatic polyamides. Ind Eng Chem Res 43: 5015-5020.

6. Cao YM, Kang GD, Gao CJ, Chen WD, Jie XM, Yuan Q (2007) Study on hypochlorite degradation of aromatic polyamide reverse osmosis membrane. J Memb Sci 300: 165-171.

7. Ettori A, Gaudichet-Maurin E, Schrotter JC, Aimar P, Causserand C (2011) Permeability and chemical analysis of aromatic polyamide based membranes exposed to sodium hypochlorite. J Memb Sci 375: 220-230.

8. Haberfie P, Paul D (1965) Chlorination of Anilines. Proof of Existence of an N-Chloro Intermediate. J Am Chem Soc 87: 5502.

9. Jones WJ, Orton KJP (1909) The chlorination of acetanilide. J Chem Soc 95 1056-1060.

10. Hardy FE, Robson P (1967) Formation and Hydrolysis of Substituted N-Chloro$\mathrm{N}$-Methylbenzamides in Aqueous Alkali. J Chem Soc B 1151-1154.

11. Hughes ED, Ingold CK (1952) Aromatic Rearrangements. Q Rev Chem Soc 6: 34-62.

12. Kucera J (2010) Reverse osmosis: design, processes, and applications for engineers, Scrivener Pub.

13. Van Thanh Do CYT, Martin Reinhard, James O. Leckie (2012) Degradation of polyamide nanofiltration and reverse osmosis membranes by Hypochlorite. Environ Sci Technol 46: 852-859. 